ARTICLE

https://doi.org/10.1038/s41467-019-13301-3

OPEN

\title{
Core architecture of a bacterial type II secretion system
}

Anastasia A. Chernyatina ${ }^{1} \&$ Harry H. Low ${ }^{1 \star}$

Bacterial type II secretion systems (T2SSs) translocate virulence factors, toxins and enzymes across the cell outer membrane. Here we use negative stain and cryo-electron microscopy to reveal the core architecture of an assembled T2SS from the pathogen Klebsiella pneumoniae. We show that 7 proteins form a $\sim 2.4 \mathrm{MDa}$ complex that spans the cell envelope. The outer membrane complex includes the secretin PuID, with all domains modelled, and the pilotin PulS. The inner membrane assembly platform components PulC, PulE, PulL, PulM and PulN have a relative stoichiometric ratio of 2:1:1:1:1. The PulE ATPase, PulL and PulM combine to form a flexible hexameric hub. Symmetry mismatch between the outer membrane complex and assembly platform is overcome by PulC linkers spanning the periplasm, with PulC HR domains binding independently at the secretin base. Our results show that the T2SS has a highly dynamic modular architecture, with implication for pseudo-pilus assembly and substrate loading.

\footnotetext{
${ }^{1}$ Department of Life Sciences, Imperial College, London SW7 2AZ, UK. *email: h.low@imperial.ac.uk
} 
$\mathrm{T}$ he bacterial T2SS is found in human pathogens such as Acinetobacter baumannii, Chlamydia trachomatis, Escherichia coli and Vibrio cholerae ${ }^{1}$. It secretes a broad repertoire of substrates including digestive enzymes and infective agents like the cholera and heat-labile (LT) toxins ${ }^{2}$. Between 12 and 15 genes in a single operon usually encode the majority of T2SS components. Whilst the soluble domains for many of these proteins have been solved by X-ray crystallography ${ }^{3,4}$, their relative stoichiometry, mode of association with binding partners, and temporal coordination for assembling a functional secretion apparatus is still poorly understood.

The protein GspD forms a 15-fold rotationally symmetric pore termed the secretin that inserts into the outer membrane $(\mathrm{OM})$ and provides a conduit for substrate into the external environment. OM insertion is usually dependent on a lipidated pilotin ${ }^{5}$, which binds to the GspD C-terminal S-domain with 1:1 stoichiometry ${ }^{6,7}$. The pilotin gene is often chromosomally discrete from the main T2SS operon. Multiple recent highresolution cryo-EM structures report the partial secretin architecture $^{8-10}$, and in complex with the pilotin ${ }^{7}$. However, the entire secretin has not yet been fully resolved due to disorder in the periplasmic N0 and N1 domains.

Within the inner membrane (IM), GspL and GspM are bitopic and monotopic membrane proteins, respectively, that together form homo- and hetero-dimers ${ }^{11}$. Combined with the polytopic membrane protein $\mathrm{GspF}^{12}$ and the ATPase GspE, these proteins form an assembly platform (AP) for the pseudo-pilus ${ }^{13}$. The pseudo-pilus constitutes a helical filament that extrudes fully folded substrate through the secretin channel. The relative stoichiometry and overall ultrastructure of the AP is unknown. GspE is a cytoplasmic AAA+ ATPase that energises the T2SS and drives pilin assembly and pseudo-pilus formation. The active state is considered to be a hexameric ring as ATP turnover is significantly upregulated in an artificially oligomerized GspE-Hcp1 fusion $^{14}$. The homologous ATPases PilB and PilT in the closely related type IV pilus (T4P) system also function as hexamers ${ }^{15,16}$. Ultimately, the functional oligomeric state and stoichiometry of GspE within the T2SS apparatus has not yet been determined. The GspE N-terminal N1E domain connects to the N2E domain with an extended linker. A shorter but known flexible linker connects the N2E domain to the C-terminal CTE ATPase domain ${ }^{17}$. Such inherent flexibility within GspE is predicted to facilitate large-scale conformational changes. The N1E domain of GspE forms a 1:1 stoichiometric complex with the cytoplasmic domain of $\mathrm{GspL}^{17,18}$. These two proteins contact $\mathrm{GspF}^{13,19}$, which is predicted to reside centrally within the AP. Concerted interplay between the GspE, GspL and GspF complex are thought crucial for coupling GspE conformational changes to the mechanical loading of pilin subunits within the pseudo-pilus assembly ${ }^{20,21}$. As GspL is a bitopic membrane protein, the direct contact between GspE and GspL also represents a mechanism for enabling cross-talk across the IM to other periplasmic components such as GspM. The coupling of the AP and outer membrane complex (OMC) across the cell envelope is mediated by GspC, where the GspC N-terminus associates with GspL and GspM within the $\mathrm{IM}^{11}$. The C-terminus of GspC must then span the periplasm as the GspC HR domain binds the GspD N0 domain $^{22}$. Despite the known interaction between GspC HR domain and GspD N0 domain, the precise arrangement of GspC HR domain at the base of the secretin remains unclear ${ }^{22}$. Similarly, understanding how GspC HR domains bind to the secretin is important for determining how the likely symmetry mismatch between $\mathrm{AP}$ and $\mathrm{OMC}$ components is overcome.

Here we isolate an assembled T2SS so that both OM and IM components are captured together. Using a fusion of cryo and negative stain EM, as well as stoichiometry measurements, we provide a reconstruction of the entire OMC and a model for the cytoplasmic components of the AP. Combined they reveal a glimpse at the core ultrastructure of this cell envelope spanning nanomachine.

\section{Results}

Purification, EM and stoichiometry of Pul ${ }_{\text {CDELMns. The T2SS }}$ from the human pathogen K. pneumoniae HS11286 strain comprises 13 genes in a single unidirectional operon termed PulC through to PulO (Fig. 1a). Note that Pul and Gsp nomenclature relate to equivalent proteins in homologous T2SS systems. The pilotin PulS is located in a separate position within the chromosome. These 14 genes were cloned and over-expressed in $E$. coli. Using affinity chromatography tags positioned on the cytoplasmic ATPase PulE and the periplasmic pilotin PulS, a complex containing seven components was purified by two successive pulldowns. Glutaraldehyde stabilisation was included after the initial pulldown. The complex comprised PulC, PulD, PulE, PulL,

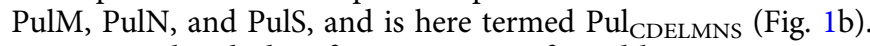
SDS-PAGE band identification was confirmed by LC-MS/MS. It is unclear why PulF and pseudo-pilus components were not copurified with Pul CDELMns. Their inclusion may require intact membrane for stabilisation. Co-expression of PulA substrate or pulG knockout did not promote their inclusion. Trace quantities of GspJ and GspK were identified on the gel by LC-MS/MS suggesting the pseudo-pilins were at least partially expressed.

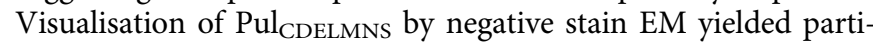
cles $\sim 40 \mathrm{~nm}$ long and 17-22 nm wide (Fig. 1c). The OMC PulD secretin was readily identifiable within $2 \mathrm{D}$ class averages. Hanging beneath the OMC and separated by a $5-10 \mathrm{~nm}$ gap the IM AP was observed. Highly flexible linkers connect the OMC and AP so that these two assemblies effectively constitute independent particles tethered together. The Pul CDELMnS complex was vitrified on thin carbon film and imaged by cryo-EM (Fig. 1c). 2D class

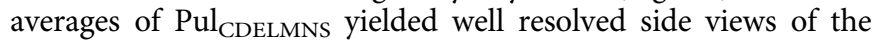
OMC. All domains of PulD were identifiable with additional densities observed at the base of the secretin where the PulC HR domain was expected to bind to the N0 domain ${ }^{22}$, and where PulS decorates the exterior of the secretin core ${ }^{7}$ (Fig. 1c). The AP was not resolved here due to high flexibility and averaging effects. In the absence of glutaraldehyde, the OMC sometimes separated from the AP and yielded top views, which confirmed PulD and PulS in 1:1 stoichiometric ratio ${ }^{7}$ with C15 symmetry (Fig. 1c). The relative stoichiometry of the IM AP components were determined by SDS-PAGE densitometry and quantification of fluorescent emission using both Coomassie R250 and Sypro Ruby dyes $^{23,24}$ (Fig. 1b). Pul C:E:L:M:N relative mean ratios standardised around PulE were observed as 2.13:1.00:0.97:0.99:0.95, which is consistent with an overall relative ratio of 2:1:1:1:1 for these components. The relative ratios of PulE and PulL were tightly correlated (sigma $=0.07$ ), which is important as it reflects their known 1:1 interaction ${ }^{17,18}$ and acts as an internal control for the gel densitometry. PulC stoichiometry was at least twice that of the other AP components with a relatively broad variance (sigma $=$ 0.19). The 15-fold copy numbers of PulD or PulS were not used as a reference to determine an overall copy number for the AP components within the Pul CDELMNS complex. PulD remained partially multimerized despite phenol treatment so that it failed to consistently enter and migrate through the gel fully. PulS quantity was significantly enriched as a consequence of the PulD secretin decoupling from the AP in the absence of glutaraldehyde stabilisation during purification.

PulD secretin structure determination. Focused refinement of the OMC yielded a reconstruction with an overall resolution of 
a

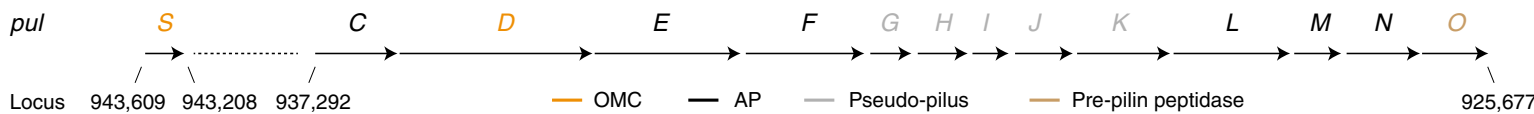

b

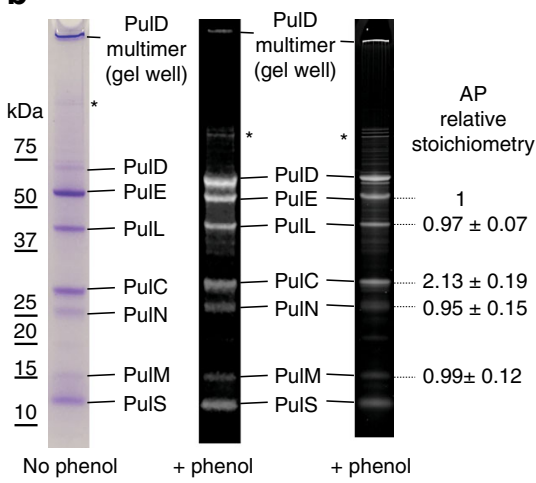

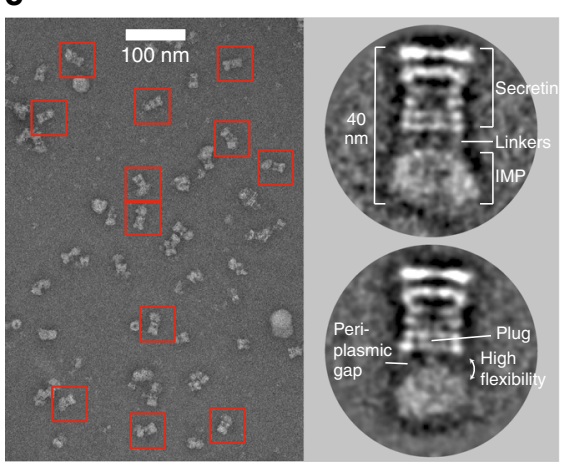

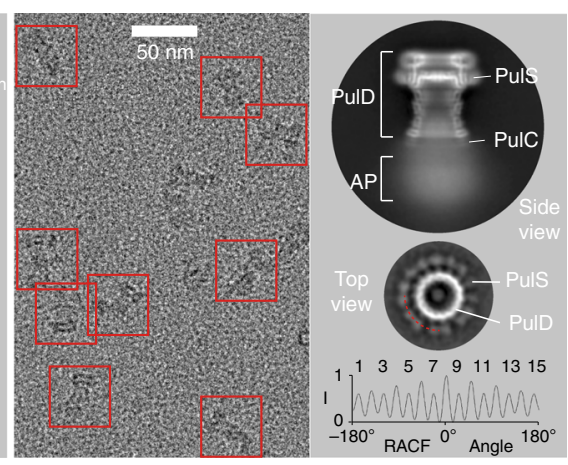

Fig. 1 Purification, stoichiometry and EM analysis of the Pul CDELMNS complex. a Schematic showing the chromosomal location and gene arrangement for the Klebsiella pneumoniae T2SS. OMC outer membrane complex, AP assembly platform. b Typical SDS-PAGE analysis of the Pul CDELMNS complex. (Left) Coomassie R250 stained gel without phenol treatment. (Middle) Fluorescent emission Coomassie R250 stained gel imaged at 680 nm with phenol extraction. Residual PuID remains in the gel well. (Right) Fluorescent emission Sypro Ruby stained gel imaged at 302 nm with phenol extraction. Relative mean stoichiometry and standard deviation for all assembly platform (AP) components are indicated. Stoichiometry measurements were determined from six independent purifications. Asterisk indicates PuID multimer. Source data are provided as a Source Data file. c Left panel shows typical negative stain EM micrograph of the Pul CDELMNS complex with selected individual particles highlighted with a red box. Zoomed images of 2D class averages are also shown. Right panel shows typical cryo-EM micrograph of the Pul CDELMNS complex with associated side view 2D class average. Top view $2 \mathrm{D}$ class average shows PulS and PuID with C15 symmetry based on the RACF. Red dotted line indicates radial ring for RACF calculation.

$4.3 \AA$ (Fig. 2 and Supplementary Fig. 1). All domains of PulD were resolved with sufficient map quality (Supplementary Fig. 2a) to build a complete model of the monomer and the secretin, excluding the amino acids (aa) in loops 288-303, 462-470 and 632-637 (Figs 3 and 4a, and Supplementary Fig. 3). The PulD fold is similar to partial E. coli K12 and H10407 GspD models (RMSD $\mathrm{Ca}=3.2 \AA$ and $3.3 \AA$ ) where the secretin core and N3 domain have been described, along with homology modelled N2 and N1 domains ${ }^{7,10}$ (Fig. 4b). The entire secretin is $20 \mathrm{~nm}$ long with an external diameter of $15 \mathrm{~nm}$ at the base (Figs $2 \mathrm{~b}$ and 3). It includes an occluding central gate and N3 domain constriction sites within the secretin channel. It lacks a $V$. cholerae cap gate ${ }^{10}$. The N1, N2 and N3 domains pack tightly (Fig. 5) with a diagonal offset of $36^{\circ}$. N0 is positioned almost directly below the N1 domain and does not maintain the diagonal offset. The N0 fold is similar to that described in multiple crystal structures $22,25,26$ with a core of two helices flanked on each side by $\beta$-sheets. However, its position relative to the $\mathrm{N} 1$ domain within the secretin is significantly different to these crystal structures where crystal contacts appear to have dominated domain arrangement (Supplementary Fig. 2b). The N0 and N1 domains are connected by loop 7 , which constitutes a substantial 26 aa linker. The Nterminus of loop 7 forms a wedge that packs between neighbouring N1 domains. Its C-terminus partially envelops the proximal N1 domain whilst making additional secondary contacts with N0 domain helix 2 (Figs 3a and 5d). The N0 domains form a tightly packed ring with alternating stacked $\beta$-sheets sandwiched between helices 2 and 4 (Fig. $5 \mathrm{~d}$ ). Failure to stabilise the N0 domain and to promote formation of the loop 7 wedge likely accounts for the previously reported N0, N1 and N2 domain flexibility in other systems ${ }^{7-10,27}$. Overall, a single PulD monomer has a radial twist around the secretin long axis of $130^{\circ}$ (Fig. 3c).

PulC HR domain binds at the secretin base. Hanging beneath the N0 domains in the OMC map, additional globular densities at
7-8 $\AA$ resolution protrude from the secretin base (Fig. 2 and Supplementary Fig. 1b). Focused refinements ${ }^{28}$ failed to markedly improve resolution. These densities were predicted to be the PulC HR domain given its known interaction with the PulD N0 domain in homologous systems ${ }^{22,29}$. Using the GspC HR domain and GspD N0 domain crystal structure 22 as a reference, a homology model of PulC HR domain was fitted as a rigid body (Supplementary Fig. 2c). The model closely follows the surface envelope of the map in this region, with a pair of triple $\beta$-sheets opposed around a central cavity (Fig. 2c). Based on the quality of this fit, the PulC HR domain was assigned to each globular protrusion. Importantly, each PulC HR domain binds to only a single PulD N0 domain and has no contact with neighbouring PulC HR domains. Overall, the binding of the PulC HR domain to the PulD N0 domain appears to be important for the correct positioning of the N0 domain within the secretin and the subsequent stabilisation of the $\mathrm{N} 1$ and $\mathrm{N} 2$ domains. Additional stabilisation is derived from a plug that occludes the lumen of the secretin at the level of the N0-N1 domains (Fig. 2b). The plug was observed in both the $2 \mathrm{D}$ class averages (Fig. 1b) and the $3 \mathrm{D}$ reconstruction. The high-resolution plug ultrastructure was not resolved due to a likely symmetry mismatch with the C15 averaged OMC. Attempts to resolve the plug structure through refinement using lower symmetries yielded reconstructions of low quality and resolution. Further studies will be required to resolve the source of the plug although the PulC PDZ domain is a speculative candidate given the position of the PulC HR domain at the base of the secretin.

The PulS pilotin decorates the secretin core. Decorating the outside of the secretin core proximal to the PulD S-domain in the map, globular densities were observed in a position consistent with the pilotin AspS relative to GspD in enterotoxigenic E. coli (ETEC) $^{7}$ (Fig. 2). For these densities, map resolution was limited to $\sim 7 \AA$ (Supplementary Fig. 1) and focused refinements ${ }^{28}$ did not markedly improve resolution. A homology model of the PulS 
a

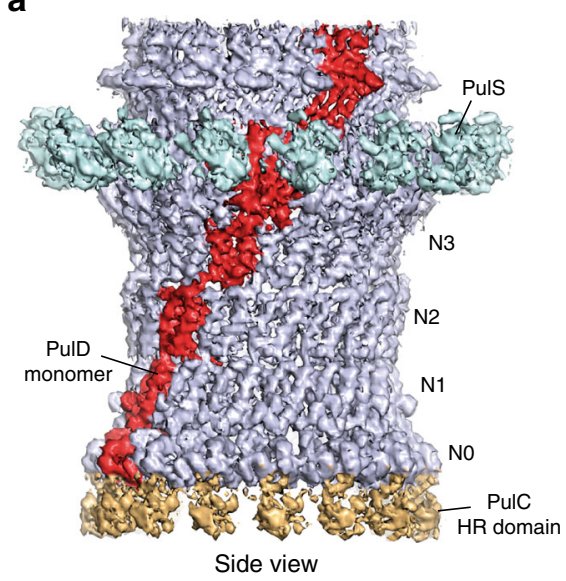

b

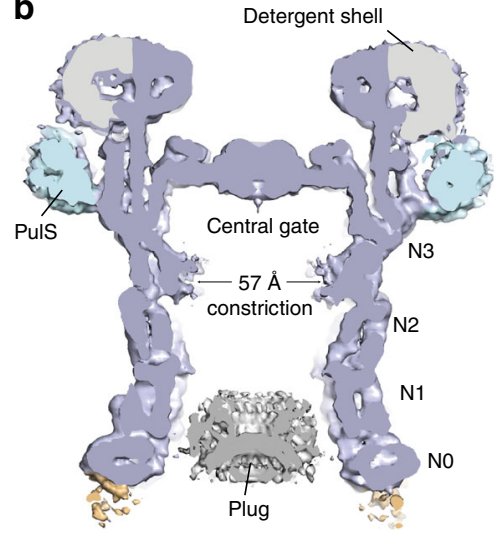

Cross section
C

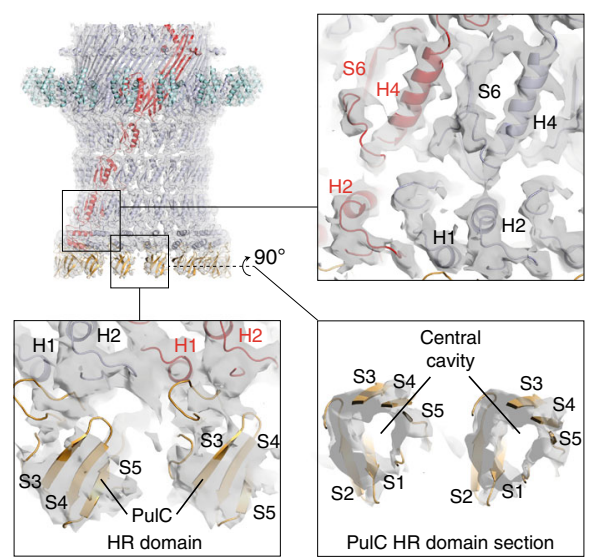

Fig. 2 Cryo-EM density map of the outer membrane complex (OMC). a $4.3 \AA$ resolution $C 15$ symmetrized cryo-EM map of the OMC locally sharpened with LocScale 48 and contoured at 5.5 . b Cross section view of the OMC revealing internal features of the PulD secretin. A plug occludes the secretin lumen. The map was unsharpened and contoured at $3 \sigma$. c Fit of molecular models within the OMC map. De novo model for the PulD secretin including the $\mathrm{N} 1$ and NO domains (top right). A PulC HR domain homology model was rigid body fitted. The map was sharpened with LocScale and contoured at $4 \sigma$

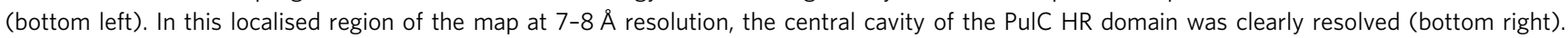

a

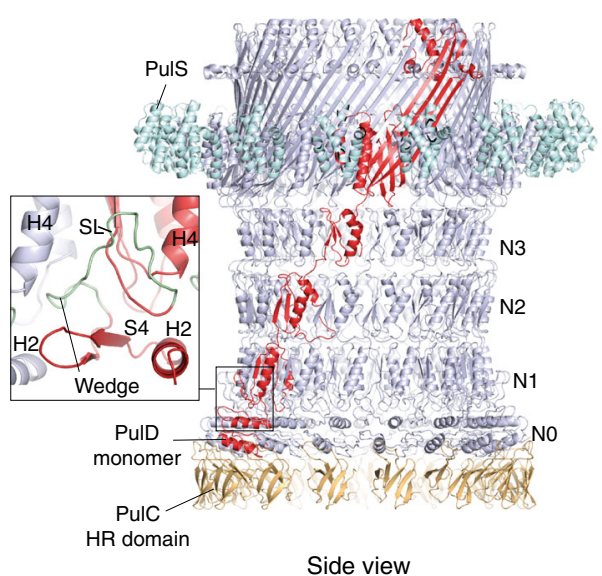

b

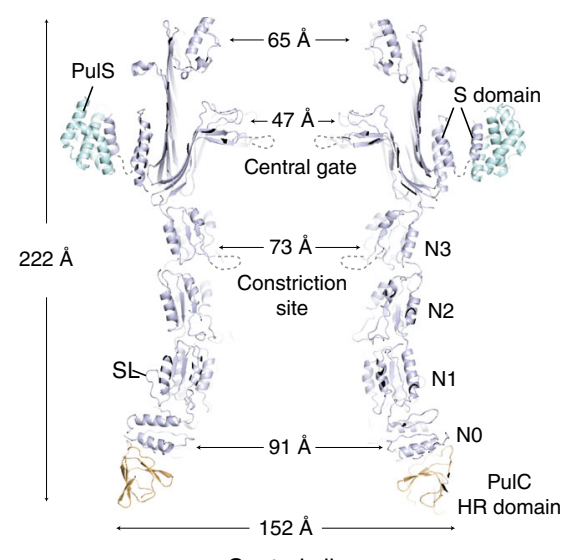

Central slice
C

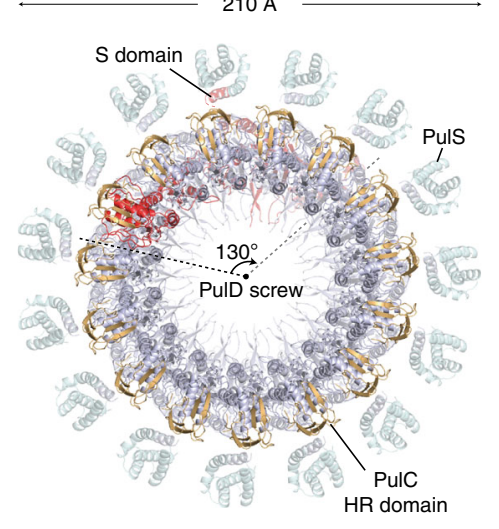

Bottom view

Fig. 3 Structure of the outer membrane complex (OMC). a Cartoon representation of the OMC, which includes the C15 symmetrized secretin (light blue) with a single PuID monomer highlighted in red. PulS (cyan) and PuIC HR domain (orange) homology models were fitted as rigid bodies. A total of 15 PulC HR domains were modelled here although stoichiometry measurements indicate an occupancy of $\sim 0.8$ in the Pul CDELMNS Complex. Zoom panel shows how loop 7 (green coil) constitutes a stabilising loop (SL) that packs as a wedge between neighbouring N1 domains. b Cross section view of the OMC structure with dimensions. c Bottom view of the OMC structure. Each PuID monomer has an azimuthal span twisting around the secretin long axis of $130^{\circ}$.

pilotin in complex with the PulD S-domain helix 15 based on the homologous structure in Dickeya dadantii (PDB $4 \mathrm{KOU}^{30}$ ) was fitted as a rigid body (Fig. 4c). Compared with $\mathrm{AspS}^{7,31}$, the position of PulS differs by a $12^{\circ}$ radial rotation around the secretin long axis (Fig. 4b). Loop 38 between S-domain helix 14 and helix 15 bound to PulS constitutes the lone contact point between the secretin core and PulS (Fig. 4c). No additional contacts were observed in contrast to AspS-GspD where the secretin core helix a11 forms extensive secondary contact with the pilotin ${ }^{7}$. The lack of equivalent secondary contacts between PulS and PulD likely accounts for the apparent flexibility between these proteins and may be a distinguishing feature between the structurally discrete Klebsiella-type and Vibrio-type pilotins.

PulC links the OMC to the inner membrane AP. Whilst the PulC HR domain binds to the base of the secretin, its $\mathrm{N}$-terminus is located within the IM AP ${ }^{11}$ so that PulC is predicted to span the periplasm and link the AP and OMC. To verify the presence and positioning of the PulC N-terminus within the AP, a hexahistidine tag was inserted after aa 61 where PulC was predicted to exit the IM and enter the periplasm. Ni-NTA gold labelling showed beads localise exclusively to the AP and not the OMC (Fig. 6a and Supplementary Fig. 4). Given the PulC HR domains bind to the base of the secretin, PulC therefore spans the periplasmic gap between the OMC and AP (Fig. 1c).

PulE, PulL and PulM form a flexible hexameric hub. Cryo-EM $2 \mathrm{D}$ class averages of the Pul CDELMNs complex revealed the ultrastructure of the AP positioned beneath the OMC. A 20-22 $\mathrm{nm}$ outer ring is coupled to a $10-12 \mathrm{~nm}$ inner ring by six radial linkers (Fig. 6b). Focused alignments of the AP where the OMC was masked out show the outer ring to be comprised of weakly associating non-contiguous globular densities (Fig. $6 \mathrm{c}$ and Supplementary Fig. 5a). This concentric ring structure is highly flexible and represents the preferred single orientation of the AP so that $3 \mathrm{D}$ structure determination was impeded. The addition of 
a

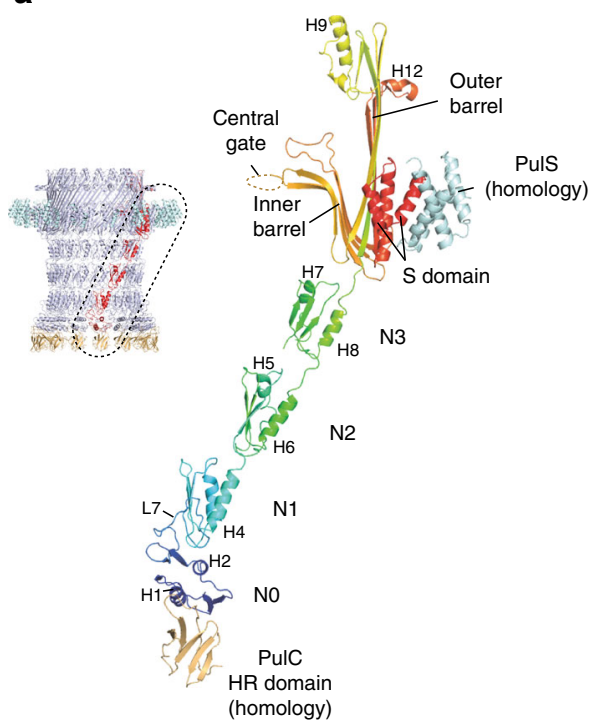

b

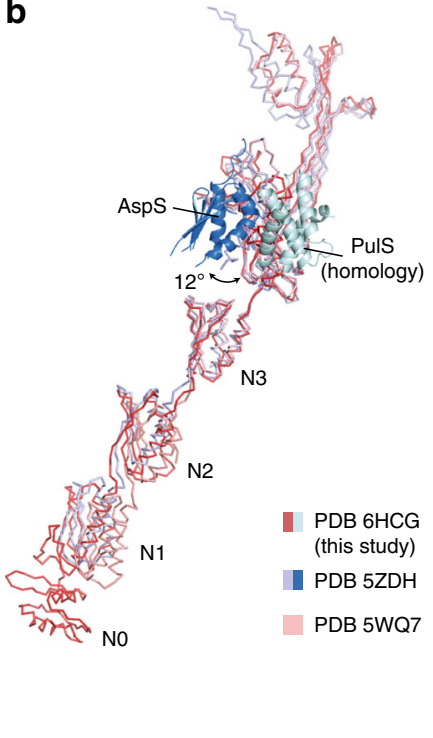

C
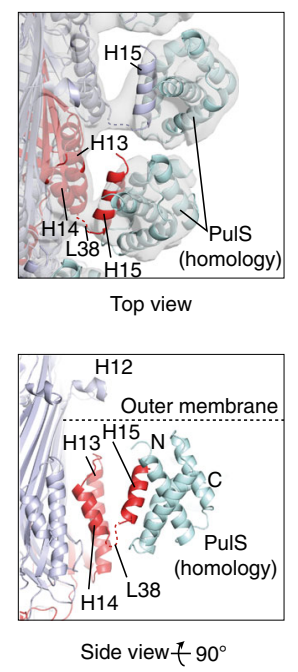

Fig. 4 Structural comparison of PuID and PulS models. a A complex of PuID, PulS and PulC HR domain monomers extracted from the C15 OMC model (dotted region). For PulD, rainbow colouring highlights blue N-terminus through to red C-terminus. $\mathbf{b}$ Superposition of the PulD and PulS complex with the equivalent from enterotoxigenic E. coli PDB 5ZDH and E. coli K12 PDB 5WQ7. Compared with PulD, these PDBs differ by RMSD C $\alpha=3.3$ and $3.2 \AA$, respectively. The relative position of AspS in PDB 5ZDH differs to PulS by a $12^{\circ}$ azimuthal rotation around the secretin long axis. $\mathbf{c}$ (Top) Fit of PulS homology model with the OMC map. The map was low pass filtered to $8 \AA$ and contoured at $5 \sigma$ before rigid body fitting. (Bottom) Cartoon schematic showing the PulS model relative to the PuID S-domain (red helices). PuID helix 15 locates to a groove within PulS 30 . Flexible loop 38 represents the only attachment between the helix 15/PulS complex and the rest of the PulD monomer.

a

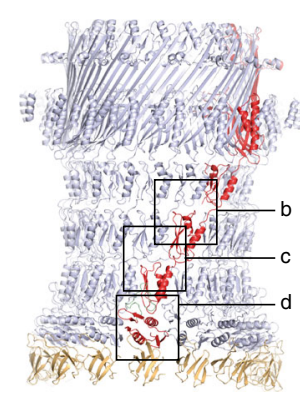

b

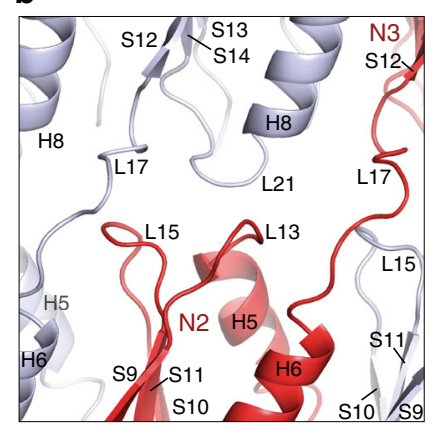

N2-N3 domain interface c

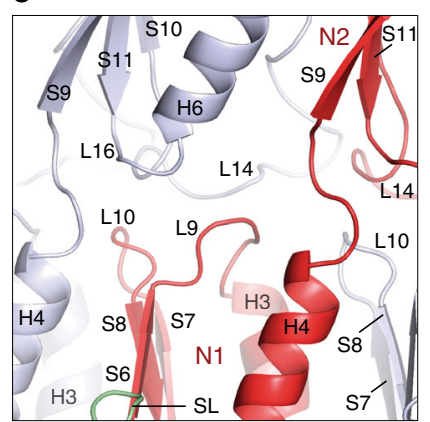

N1-N2 domain interface

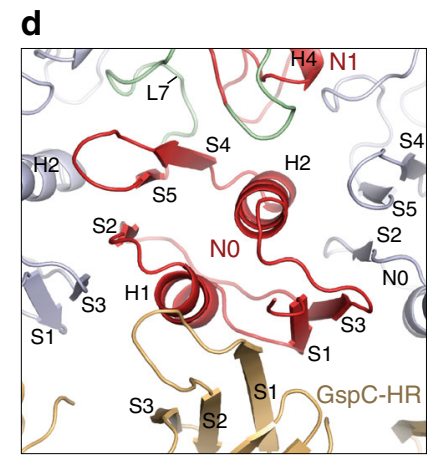

PulC HR-N0-N1 domain interface

Fig. 5 Structural analysis of the outer membrane complex (OMC) periplasmic domains. a Overview model of the OMC with PulS removed for clarity. Boxed regions are zoomed in b-d. b-d Cartoon representation of selected domain interfaces showing key structural details.

non-hydrolysable ATP analogues made no obvious change to the Pul CDELMns complex under conditions tested. In order to dissect the observed AP ultrastructure, a sub-assembly constituting PulE, PulL, PulM and PulN (Pul $\left.\mathrm{ELMN}_{\mathrm{N}}\right)$ was purified by two-step affinity chromatography. GraFix ${ }^{32}$ stabilised the complex and reduced particle heterogeneity. PulN bound weakly to the complex and only trace quantities were observed by SDS-PAGE within the Pul $_{\text {ELMn }}$ complex after GraFix (Supplementary Fig. 5b). The resultant Pul $\mathrm{ELM}_{\mathrm{M}}$ complex was analysed by cryo and negative stain EM on continuous carbon film (Fig. 6d, e and Supplementary Fig. 5b, c). Under vitreous conditions, Pul $_{\text {ELM }}$ yielded a preferred orientation concentric ring structure that was similar to the AP within the Pul CDELMNs complex, with equivalent dimensions and overall C6 symmetry. By negative stain, the same ultrastructure was observed although the sample was compacted so that the outer and inner rings have dimensions of $16-18$ and $8-9 \mathrm{~nm}$, respectively. Compaction was likely a consequence of sample flexibility and drying effects during the negative stain procedure. PulE alone, and PulE in complex with the cytoplasmic domain of
PulL (aa 1-235) or full-length PulL were also purified from the membrane fraction. However, EM analysis yielded heterogeneous and flexible particles that did not further resolve AP ultrastructure under conditions tested.

\section{Discussion}

Negative stain and cryo-EM studies of the Pul CDELMnS $_{\text {complex }}$ revealed the ultrastructure of the AP positioned beneath the OMC to be a highly flexible hexameric hub comprising two concentric rings. In vitreous ice, the inner ring had a diameter of $\sim 11 \mathrm{~nm}$ and overall C6 symmetry. The outer ring constituted non-contiguous globular densities with $\sim 22 \mathrm{~nm}$ diameter. Similar ultrastructure was observed with purified Pul $\mathrm{ELM}_{\text {complex }}$ meaning just these components alone are sufficient to generate the bulk of the hexameric hub structure. In both systems, the single preferred orientation captured was considered to represent an end view projection of the AP. In the T2SS, the V. cholerae PulE homologue GspE forms a quasi C6 ring with diameter 
a

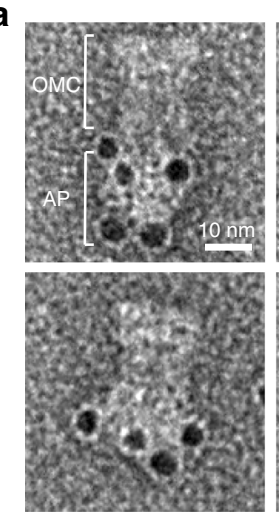

d

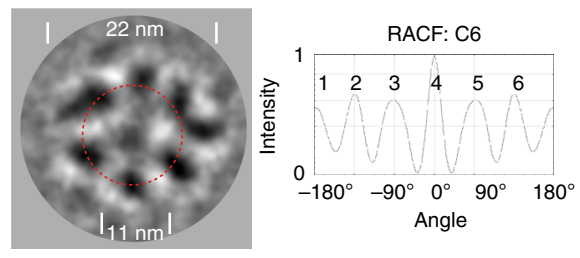

e

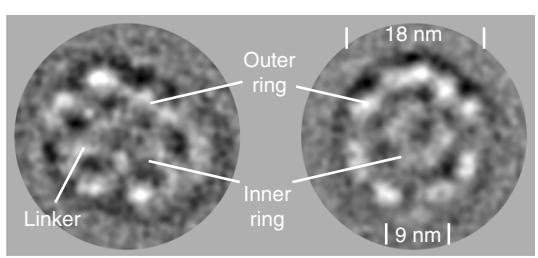

f

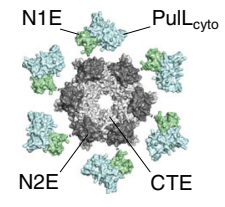

End view
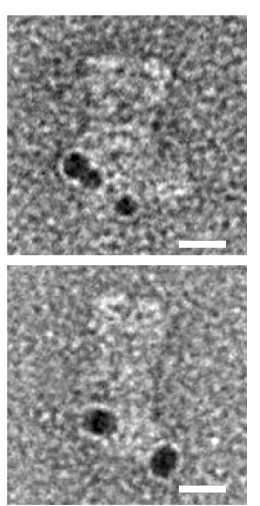

b

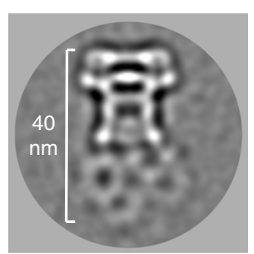

g

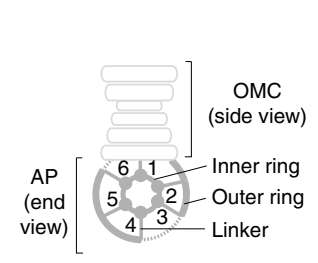

C
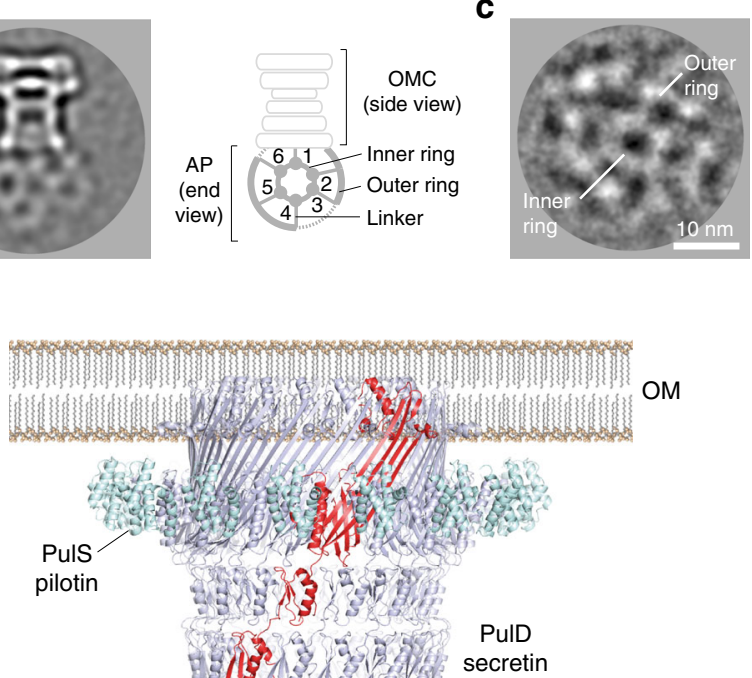

OM

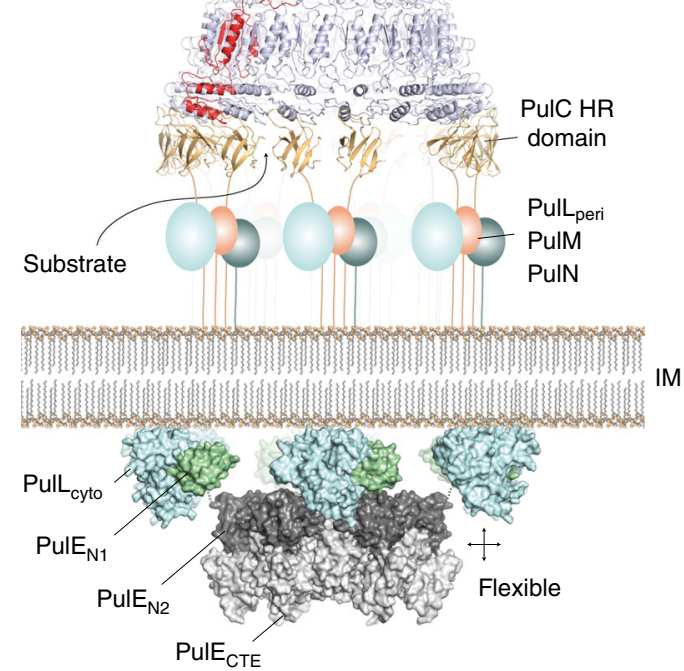

Fig. 6 2D EM of the assembly platform (AP) and a T2SS core model. a Ni-NTA gold bead labelling of the PulC N-terminus. Beads locate exclusively to the AP. $\mathbf{b}$ Cryo-EM 2D class average of Pul CDELMNs. The AP showed end views as a preferred orientation with C6 symmetry. $\mathbf{c}$ Cryo-EM 2D class average of Pul CDELMns with the alignment and classification focused on the AP. d Cryo-EM 2D class average of Pul ELM showing C6 symmetry. RACF rotation autocorrelation function calculated around dotted red radial ring. e Negative stain EM 2D class average of Pul $\left.\right|_{\text {ELM. }}$. Schematic showing modelled arrangement of the Pul EL cytoplasmic domains within the Pul CDELMNS and Pul ELM hexameric hub. The central PulE hexamer (inner ring, PDB 4KSS) is decorated by six copies of the Pul $\mathrm{E}_{\mathrm{N} 1 \mathrm{E} / \mathrm{Lcyto}}$ complex (outer ring, PDB 2BH1). Connection between inner and outer ring is mediated by the 44 aa flexible N1E-N2E interdomain linker. $\mathbf{g}$ Stoichiometric model of an assembled T2SS core apparatus. AP components comprise a hexameric hub. PulC connects the AP to the OMC by binding to the secretin base. Substrate may be loaded through the PulC cage in positions where GspC is absent.

11-13 nm when combined with an Hcp1 fusion ${ }^{14}$. The related T4P system is energised by the ATPases PilB and PilT, which are known to operate as highly dynamic hexameric rings. PilB has dimensions of 13.5 and $9 \mathrm{~nm}$ when in an elliptical C2 conformation ${ }^{15}$ whilst the PilT ring has a diameter of $11.5 \mathrm{~nm}^{33}$. For PilB and GspE these dimensions are specific to the N2E and CTE ATPase domains and do not include their N-terminal N1E domains. In situ reconstruction of the T4P system in Myxococcus xanthus indicates the position of PilB and PilT to be located centrally at the base of the $\mathrm{AP}^{34}$. Collectively, this data is consistent with a model for the Pul $\mathrm{CDELMNS}_{\mathrm{AP}}$ and Pul $\mathrm{PLM}_{\mathrm{EL}}$ complex in which the observed inner ring constitutes a PulE hexamer comprising the N2E and CTE domains similar to that observed in V. cholerae $\mathrm{GspE}^{14}$ (Fig. 6f).

The $V$. cholerae GspE N1E domain forms a direct 1:1 stoichiometric complex with the cytoplasmic domain of GspL ${ }^{17,18}$. The equivalent domains in $K$. pneumoniae also form a 1:1 stoichiometric complex termed here Pul $\mathrm{E}_{\mathrm{N} 1 \mathrm{E} / \mathrm{Lcyto}}$ (Supplementary
Fig. 5d). In the T4P system, the PulL cyto $_{\text {homologue PilM forms a }}$ ring around the central PilB/PilT hexamer. This architecture supports a model for the Pul CDELMnS AP and Pul ${ }_{\text {ELM }}$ complex where the bulk of the hexameric hub outer ring constitutes $\mathrm{Pul}_{\mathrm{E}-}$ N1E/Lcyto complexes (Fig. 6f). PulM, the periplasmic domain of PulL (termed PulL $\mathrm{L}_{\text {peri }}$ ), and PulN and PulC when present, may also contribute to the observed outer ring densities if ordered.

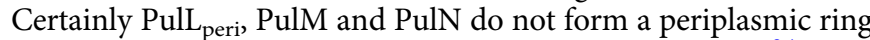
here as might be expected based on the T4P system ${ }^{34}$. The retention of membrane or inclusion of other T2SS components such as the pseudo-pilus may be required for the stabilisation and visualisation of any such ring.

Within the Pul CDELMns complex, the mean relative ratio of PulC: PulE: PulL:PulM:PulN was measured to be 2.13:1.00:0.97:0.99:0.95 indicating two copies of PulC for each of the AP components. The globular densities that comprise the outer ring of the hexameric hub do not form a contiguous well-ordered structure. This observation combined with the stoichiometry supports a modular architecture 
where independent or weakly associated sub-complexes containing two copies of PulC and a single copy of PulM, PulN and Pul $\mathrm{E}_{\mathrm{N} 1 \mathrm{E}}$ in complex with PulL decorate each subunit of the central PulE hexamer (Fig. 6g). This model does not preclude the possible formation of a Pul $\mathrm{E}_{\mathrm{N} 1 \mathrm{E} / \mathrm{Lcyto}}$ ring positioned more centrally directly above the PulE N2E/CTE hexamer in vivo. Such an arrangement would be similar to that observed for the N1 domains of Thermus thermophilus PilF $\mathrm{F}^{35}$ and may promote the close contact and encircling of the putative pilin spooling protein $\operatorname{Pul}^{13,19}$.

PulC spans the periplasm to bind the OMC with the PulC Nterminus located within the IM and the HR domain bound to the PulD N0 domain at the secretin base. Based on six PulE subunits within the AP, stoichiometry measurements support a model where the PulC copy number within the AP is 12 . PulC therefore constitutes a cage that spans the periplasm (Fig. 6g) and is reminiscent of the virB10 $\mathrm{N}$-terminus which spans the periplasm in the type IV secretion system ${ }^{36}$. Substrate may have the potential to gain access to the secretin lumen via PulL and PulM ${ }^{37}$ in positions where PulC is absent. The OMC structure shows that each PulC HR domain binds a single PulD No domain independently with no lateral contacts between neighbouring HR domains. This is important as it provides a natural mechanism for overcoming symmetry mismatch between the 12 PulC subunits located within the hexameric AP and the 15 -fold symmetric OMC. In this model on average three secretin No domains remain unoccupied without bound PulC HR domain. Whether full secretin N0 domain binding occupancy occurs with up to 15 bound PulC HR domains cannot be entirely excluded as each of the No domains has at least the potential to bind a PulC HR domain. T2SS assembly and secretion may ultimately occur in a dynamic equilibrium with a range of N0 domain binding occupancies.

Multiple T2SS secretin structures have been solved including Klebsiella-types from E. coli $\mathrm{K}_{1} 2^{10}$ and Pseudomonas aeruginosa ${ }^{9}$, and Vibrio-types from ETEC 7 , enteropathogenic E. coli $i^{8}$ and $V$. cholerae $^{10}$. Despite high quality 3-3.5 $\mathrm{A}$ resolutions achieved for the secretin core and N3 domains, all the structures have poorly resolved densities towards the $\mathrm{PulD} / \mathrm{GspD} \mathrm{N}$-termini with homology modelling required for the N1 domains and entirely absent N0 domains. Docking of N0 and N1 domain co-crystal structures $^{22,25-27}$ into the secretin maps failed to resolve the possible position and orientation of the N0 domain due to significant steric clashes. Consequently, the PulC HR domain orientation relative to the No domain remained unclear and it was modelled decorating the inside of the secretin base ${ }^{22}$. To account for the high flexibility of the secretin N0 and N1 domains and the symmetry mismatch between the sixfold symmetric AP and 15-fold OMC, a pseudo-sixfold arrangement comprising a hexamer of dimers had been proposed for the N0-N2 domains 9 . In such a model the N0-N2 domains of three secretin subunits would be displaced in a metastable arrangement. The OMC structure derived from the Pul CDELMNs complex resolves now how the PulD N0 domains are organised within the secretin relative to the $\mathrm{N} 1$ domain ring. The $\mathrm{N}$-terminus of loop 7 wedges and stabilises neighbouring $\mathrm{N} 1$ domains whilst the N0 domains form an intimate and stable C15 ring. At least when PulC HR domain is bound, the N0 ring does not immediately support a model where the secretin incorporates multiple symmetries along its length. Instead the secretin forms a well-ordered barrel, which combined with the PulC HR domains bound at the base protrudes from the outer membrane $\sim 20 \mathrm{~nm}$ into the periplasm.

In this study, the T2SS outer membrane components PulD and PulS are co-purified with the core components of the IM AP including PulC, PulE, PulL, PulM and PulN. Overall, our results reveal a glimpse at the core architecture of an assembled T2SS (Fig. $6 \mathrm{~g}$ ) and show it to be different to other known secretion systems (Supplementary Fig. 6). The T2SS AP does not constitute a stack of conjoined rings that seal directly to the secretin base forming an enclosed central channel as observed in the T3SS ${ }^{38}$. Instead, the T2SS has a highly dynamic and modular architecture where the OMC and IM AP have weakly associating and limited inter-connection via a PulC cage. This observation is consistent with the requirement for substrate to gain access to the secretin lumen via the periplasm. Whilst the resolution of the hexameric hub observed in the Pul $\mathrm{CDELMNS}_{\text {and }} \mathrm{Pul}_{\mathrm{ELM}}$ complexes is limited it shows the T2SS to have a sixfold arrangement for assembled AP components. The observed AP architecture is consistent with that described in the T4P system ${ }^{34}$ providing evidence that the IM ultrastructure is broadly conserved between these different but related secretion systems. Although the connection between inner and OMCs was expected to be transient and dynamic ${ }^{3}$, we show that it is possible to isolate an almost fully assembled T2SS in vitro. Our work suggests that the purification of an entire T2SS with the pseudo-pilus, PulF, and ultimately substrate incorporated is in reach. These additional components may be necessary to help stabilise the IM AP so that ultimately full structural dissection and mechanistic understanding for the T2SS may be achieved.

\section{Methods}

Cloning, protein expression and purification. All clones were generated using a modified version of the Gibson isothermal DNA assembly protocol ${ }^{39}$ where the Taq DNA ligase was omitted from the one-step isothermal DNA assembly. To

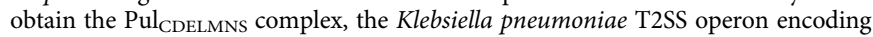
genes from pulC to pulO was cloned into pASK3c vector (IBA-GO) using primer pair 1 and 2 (for all primers used see Supplementary Table 2). A StrepII tag was added at the C-terminus of PulE using primer pair 3. The pulS gene was cloned into pCDF-duet vector using primer pair 4 and 5. A C-terminal Flag tag was inserted using primer pair 6. Uniprot codes for individual genes are as follows: pulC KPHS_08880, pulD KPHS_08870, pulE KPHS_08860, pulF KPHS_08850, pulG KPHS_08840, pulH KPHS_08830, pulI KPHS_08820, pulJ KPHS_08810, pulK KPHS_08800, pulL KPHS_08790, pulM KPHS_08780, pulN KPHS_08770, pulO KPHS_08760 and pulS KPHS_08940. The clones were co-transformed into E. coli C43 (DE3) electro-competent cells (Lucigen) modified here to incorporate a $p s p A$ gene knockout using a Lambda Red recombinase strategy ${ }^{40}$ (PspA is a common contaminant induced by PulD over-expression). Cells were grown on selective LBagarose plates with chloramphenicol $(30 \mu \mathrm{g} / \mathrm{ml})$ and spectinomycin $(50 \mu \mathrm{g} / \mathrm{ml})$. $2 \times \mathrm{YT}$ media was inoculated and cells grown at $37^{\circ} \mathrm{C}$ until induction at $\mathrm{OD}_{600}=$ $0.5-0.6$ with anhydrotetracycline (AHT, $0.2 \mathrm{mg} / \mathrm{L}$ ) and isopropyl $\beta$-D-1thiogalactopyranoside (IPTG, $0.24 \mathrm{~g} / \mathrm{L}$ ). Cells were grown for $\sim 15 \mathrm{~h}$ at $19{ }^{\circ} \mathrm{C}$ and processed immediately. Pellets were re-suspended in ice-cold buffer $50 \mathrm{mM}$ Tris$\mathrm{HCl} \mathrm{pH} \mathrm{7.5,} 5 \mathrm{mM}$ EDTA, treated with DNase I, lysozyme and sonicated on ice. The lysate was clarified by centrifugation at $16,000 \times g$ for $20 \mathrm{~min}$. The membrane fraction was collected by centrifugation at $142,000 \times g$ for $45 \mathrm{~min}$. Membranes were mechanically homogenised and solubilized in $50 \mathrm{mM}$ Hepes- $\mathrm{NaOH} \mathrm{pH}$ 7.5, $150 \mathrm{mM} \mathrm{NaCl}, 1 \% \mathrm{w} / \mathrm{v}$ DDM (Anatrace) and $5 \mathrm{mM}$ EDTA at room temperature for $30-40 \mathrm{~min}$. The suspension was clarified by centrifugation at $132,000 \times g$ for $15 \mathrm{~min}$. The supernatant was loaded onto a StrepTrap HP column (GE Healthcare) and washed with $50 \mathrm{mM}$ Hepes- $\mathrm{NaOH}$ pH 7.5, $150 \mathrm{mM} \mathrm{NaCl}, 0.06 \%$ w/v DDM and $5 \mathrm{mM}$ EDTA (Buffer W) at $4{ }^{\circ} \mathrm{C}$. All prior buffers were supplemented with EDTA-free complete protease inhibitor tablets (Roche). The protein sample was eluted in Buffer W supplemented with $2.5 \mathrm{mM}$ desthiobiotin (IBA) but with protease inhibitors removed. Peak fractions were pooled, $0.05 \%$ glutaraldehyde

(Sigma-EM grade) added and incubated on ice for $10 \mathrm{~min}$ before quenching with $100 \mathrm{mM}$ Tris-HCl pH 7.5. The sample was batch incubated with Flag resin (Sigma) for $1 \mathrm{~h}$. Flag resin was washed with Buffer W and then eluted with the same buffer supplemented with $3 x$ Flag peptide. Peak fractions were collected and used immediately. LC-MS/MS confirmed the identity of bands identified by SDS-PAGE

To obtain purified Pul ${ }_{\text {ELMN }}$ complex, the full-length pulE gene was cloned into pASK3c vector to include a C-terminal StrepII tag (primer pair 7 and 8). The pulL, pulM and pulN region of the T2SS operon was cloned into PCDF-duet vector with a PulN C-terminal Flag tag (primer pair 9 and 10). These vectors were cotransformed and the same initial purification strategy was then followed as for the Pul CDELMNS complex with the exception that no glutaraldehyde or Tris quenching buffer were added subsequent to elution from the Strep column. Protease inhibitor tablets were included in all buffers. After elution from the Flag column, due to sample heterogeneity as judged by negative stain $\mathrm{EM}, \mathrm{GraFix}^{32}$ was undertaken. Using Beckman Ultra-Clear $4.2 \mathrm{ml} 11 \times 60 \mathrm{~mm}$ ultracentrifugation tubes $2.1 \mathrm{ml}$ of $50 \mathrm{mM}$ Hepes- $\mathrm{NaOH}, 150 \mathrm{mM} \mathrm{NaCl}, 0.06 \% \mathrm{w} / \mathrm{v}$ DDM, $30 \%$ v/v glycerol, $5 \mathrm{mM}$ EDTA and $0.1 \%$ glutaraldehyde was loaded under $2.1 \mathrm{ml}$ of the equivalent but with $10 \% \mathrm{v} / \mathrm{v}$ glycerol. A continuous gradient was made using a BioComp Gradient 
Master cycle set for $66 \mathrm{~s}$ at $83^{\circ}$ tilt and $22 \mathrm{rpm}$. An equivalent gradient was also made but omitting glutaraldehyde so that the sample could be analysed by SDSPAGE after centrifugation. The Pul $\mathrm{PLMN}_{\text {sMmple }}$ sas split in half and loaded onto the gradients \pm glutaraldehyde. Samples were spun at $71,000 \mathrm{~g}$ for $16 \mathrm{~h}$ at $4{ }^{\circ} \mathrm{C}$ using a Beckman Sw $60 \mathrm{Ti}$ swing rotor. $150 \mu$ fractions were collected manually and analysed. Note that PulN was generally lost from the Pul $\mathrm{ELMN}_{\mathrm{N}}$ complex during GraFix yielding just $\mathrm{Pul}_{\text {ELM }}$.

To obtain purified Pul $\mathrm{E}_{-\mathrm{N} 1 \mathrm{E} / \mathrm{Lcyto}}$ complex, the N1E domain comprising aa 1-108 from pulE was cloned into pASK3c vector to include a C-terminal StrepII tag (primer pair 8 and 11). For Pul $\mathrm{L}_{\text {cyto }}$ aa 1-235 relating to the cytoplasmic domain of PulL were cloned into pCDF-duet vector with a C-terminal Flag tag (primer pair 10 and 12). The same two-step affinity chromatography purification strategy was then followed as for PulELMN but excluding Grafix. Note that the Pul $\mathrm{E}_{\mathrm{E} \text {-NE/Lcyto }}$ complex readily purifies from the membrane fraction despite the removal of the PulL trans-membrane domain.

Gold labelling. This was performed on the Pul $\mathrm{CDELMNS}_{\text {complex modified to }}$ include a hexahistidine tag within PulC after aa 61 (primer pair 13). Purification was the same as for the Pul CDELMNS complex but without the addition of fixative. Protease inhibitor tablets were included in all buffers. Ni-NTA-Nanogold (Nanoprobes) pre-washed in Buffer $\mathrm{W}$ was added to $10 \mu \mathrm{l}$ of the protein sample and incubated for $30 \mathrm{~min}$ at $4{ }^{\circ} \mathrm{C}$. A homemade continuous carbon grid was deposited on the $10 \mu \mathrm{l} \mathrm{sample}$ for $3 \mathrm{~min}$, blotted and washed two times in Buffer $\mathrm{W}$ supplemented with $10 \mathrm{mM}$ imidazole, then two times in Buffer $\mathrm{W}$ before being stained with three drops of $2 \%$ uranyl acetate.

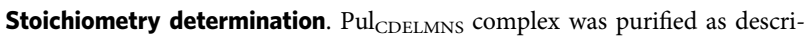
bed above with the exception that no glutaraldehyde or Tris quenching buffer were added subsequent to elution from the Strep column. Protease inhibitor tablets were included in all buffers. Six independent purifications were extracted using phenol to disrupt PulD multimerization ${ }^{41}$. Before extraction samples were divided and phenol treated in duplicate. The samples were precipitated with an equal volume of phenol and immediately vortexed. Four volumes of ice-cold acetone were then added and vortexed again. The mixture was kept overnight at $-20^{\circ} \mathrm{C}$. The precipitate was pelleted in a bench-top centrifuge at $14,000 \times g$ for $30 \mathrm{~min}$ at $4^{\circ} \mathrm{C}$, washed once with ice-cold acetone, dried under vacuum and re-suspended in loading buffer supplemented with $10 \mathrm{mM}$ DTT. Both duplicates for each independent purification were analysed by SDS-PAGE densitometry and quantification of fluorescent emission using both Coomassie Blue R250 and Sypro Ruby dyes. The Coomassie R250 procedure was as described ${ }^{24}$ but with extended stain and destaining times. Briefly, gels were rinsed with water and then stained with $0.01 \%$ Coomassie Blue R250 in 50\% methanol and 10\% acetic acid for $20 \mathrm{~min}$. Gels were rinsed with $40 \%$ methanol and $7 \%$ acetic acid and then destained twice with the same solution for $20 \mathrm{~min}$ each time. Finally, gels were soaked 2-3 times in water until fully destained, usually $1-2 \mathrm{~h}$. Gels were imaged on a Licor Odyssey $\mathrm{Fc}$ at $680 \mathrm{~nm}$. Sypro Ruby gel staining was undertaken following the manufacturer's instructions (Bio-Rad). Gels were imaged on a Bio-Rad Chemidoc XRS imaging system at $302 \mathrm{~nm}$. Image Lab software (Bio-Rad) and ImageJ were used to calculate band intensities. Uncropped gels are provided as a Source Data file.

To determine the stoichiometry of the Pul $\mathrm{E}_{\mathrm{N} 1 \mathrm{E} / \mathrm{Lcyto}}$ complex, two independent purifications were undertaken. Samples were run in duplicate on gels and then stained using the Coomassie Blue R250 procedure as outlined above. Gels were imaged on a Licor Odyssey Fc at $680 \mathrm{~nm}$.

\section{Electron microscopy sample preparation and data collection. For OMC} structure determination, $4 \mu \mathrm{l}$ of purified Pul CDELMNs complex solution was incubated for $30 \mathrm{~s}$ on glow discharged homemade continuous thin carbon grids before vitrification in liquid ethane using a Vitrobot Mark IV (FEI). Data were collected at $300 \mathrm{kV}$ on a Titan Krios (M02 beamline at eBIC Diamond, UK) equipped with a Gatan Quantum K2 Summit detector. Images were acquired at a magnification of $\times 28,090$ yielding $1.78 \AA /$ pixel using EPU software. Images were dose-weighted over 40 frames with $12 \mathrm{~s}$ exposures. Total dose was $\sim 50 \mathrm{e} / \AA^{2}$.

All other cryo and negative stain datasets were collected in-house at $200 \mathrm{kV}$ on a Tecnai F20 microscope equipped with Falcon II direct electron detector. For cryo data, Pul ${ }_{\text {CDELmns }}$ complex (Fig. 6b, c and Supplementary Fig. 5a) was vitrified as

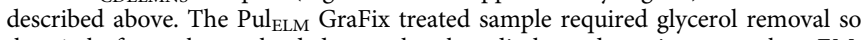
that $4 \mu \mathrm{l}$ of sample was loaded onto the glow discharged continuous carbon EM grid and after 1-min incubation was washed four times in Buffer $\mathrm{W}$ before plunge freezing. Images were acquired at a magnification of 90,909 yielding $1.65 \AA /$ pixel using EPU software. Images were collected over 54 frames with $3 \mathrm{~s}$ exposures. Total dose was $\sim 50 \mathrm{e} / \AA^{2}$. For negative stain data, $4 \mu \mathrm{l}$ of Pul CDELMNS complex was loaded onto the glow discharged continuous carbon EM grid, after $40 \mathrm{~s}$ the grid was washed with 3 drops of distilled water and stained with 3 drops of $2 \%$ uranyl

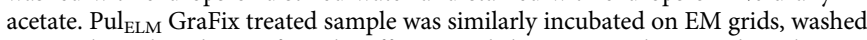
iteratively with 4 drops of $15 \mu$ l Buffer $\mathrm{W}$ and then negatively stained as above. Images were acquired at a magnification of 90,909 using EPU software. Single frames were collected with $1 \mathrm{~s}$ exposure and total dose $\sim 15 \mathrm{e} / \AA^{2}$.
Image processing. For OMC structure determination, individual movie image frames were aligned with MotionCor2 $2^{42}$ and the contrast transfer function estimated using Gctf $1.06^{43}$. Low quality images were discarded and 3427 micrographs used for subsequent reconstruction in Relion $2.1^{44}$. Initial manual particle picking

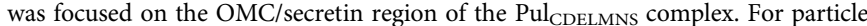
extraction a box and mask diameter were chosen so that contributions from the IM AP were excluded. In this way, low-resolution $2 \mathrm{D}$ class averages of just the OMC were used as a template for autopicking. OMC side views only were prevalent in this dataset, which provided a sufficiently even equatorial band distribution for a reliable reconstruction ${ }^{45}$. Low quality particles were removed by four rounds of $2 \mathrm{D}$ classification resulting in a stack of 36,240 particles. A single round of 3D classification was undertaken generating ten classes. The V. cholerae GspD reconstruction EMDB-1763 was used as an initial model filtered to $40 \AA^{46}$ C15 symmetry was applied based on top views of the OMC (obtained in an alternative Pul CDELMNS $_{\text {complex purification) and an unambiguous } 15 \text { peaks }}$ observed from the rotation auto-correlation function calculation (Fig. 1c). A single class containing 7284 particles was used for the final refinement, which attained $4.4 \AA$ resolution. Post-processing yielded $4.3 \AA$ resolution with an auto-estimated B-factor ${ }^{47}$ of $-142 \AA^{2}$ applied to sharpen the final 3D map for model building. A locally sharpened map was also generated using LocScale ${ }^{48}$ once initial models were built. Further particle polishing and 3D refinement did not yield a marked increase in resolution. Resolutions reported are based on gold standard Fourier shell correlations $(\mathrm{FSC})=0.143$. Statistics for data collection and 3D refinement are included in Supplementary Table 1. Local 3D refinements with various particle subtraction strategies focusing on PulS or PulC HR domain did not markedly improve resolution.

To generate all other 2D class averages both in cryo and negative stain conditions as for Pul $\mathrm{CDELMNS}_{\text {and }}$ and $\mathrm{Pul}_{\mathrm{ELM}}$ complexes, the following protocol was followed. Working initially within Relion 2.0 or 2.1, Gctf 1.06 was used for estimating the CTF. Negative stain micrographs were phase flipped. Low quality micrographs were discarded. Initial $2 \mathrm{D}$ class averages were generated from a manually picked stack to yield templates for autopicking. A total of 3-4 rounds of 2D classification were then undertaken to remove low quality particles. Using the 'relion_stack_create' a cleaned image stack was generated for further processing. For cryo-EM images the stack was created from phase flipped particles. In Imagic ${ }^{49}$, particles were normalised, band pass filtered, centred and subjected to reference-free MSA and classified. The best classes, typically judged by lowest variance, were used as references for multi-reference alignment (MRA) in Spider ${ }^{50}$ followed by MSA and classification in Imagic. This cycle of MRA and MSA was typically iterated a further 2-3 times. For negative stain Pul $\mathrm{ELM}_{\text {M }}$ data, 2746 micrographs yielded 81,727 extracted particles and a cleaned stack of 63,014 particles. For cryo Pul $\mathrm{ELM}_{\mathrm{LM}}$ data, 467 micrographs yielded 4937 hand-picked particles, and all particles were used for subsequent MSA and MRA. For Pul CDELMNS $_{\text {, }} 3059$ micrographs yielded 89,381 extracted particles and a cleaned stack of 66,855 particles for subsequent MSA and MRA (Fig. 6b). From this same dataset, 1148 micrographs were then used to hand-pick $4020 \mathrm{AP}$-focused particles (Fig. $6 \mathrm{c}$ and Supplementary Fig. 5a). Rotation auto-correlation functions were calculated using Imagic.

Model building. A PulD homology model was generated with I-Tasser ${ }^{51}$ using the E. coli GspD PDB 5WQ7 as a template. This yielded a starting model for the secretin core, and N3, N2 and N1 domains. E. coli GspD shares $57 \%$ sequence identity with PulD. A homology model for the N0 domain was generated using Swissmode $l^{52}$ with the relevant part of 3 OSS as a template (51\% sequence identity). Homology models were rigid body fitted into the map using Chimera ${ }^{53}$ Fit in Map function. Using these models as a starting guide and the side chain detail from bulky residues to confirm sequence register, $\operatorname{Coot}^{54}$ was used to manually build a complete model for PulD aa 27-652 excluding aa 288-303, 462-470 and 632-637. The model was further refined using real-space refinement in Phenix ${ }^{55}$ with secondary structure, geometry and NCS restraints applied. For the low-resolution regions specific to the PulC HR domain, a homology model based on ETEC GspC HR domain PDB 3OSS (27\% sequence identity) was generated using Swissmodel. For fitting this homology model, the PDB 3OSS which includes the ETEC GspD N0 domain was first superimposed onto the PulD N0 domain. The PulC HR domain homology model was then superimposed onto the ETEC GspC HR domain from PDB 3OSS resulting in a near perfect fit within the map. The Chimera Fit in Map function was then applied to PulC HR domain resulting in a minor shift so that the PulC HR-PulD N0 domain complex has a RMSD Ca = $1.4 \AA$ when aligned to PDB 3OSS (Supplementary Fig. 2c). For the low-resolution regions specific to the PulD S-domain C-terminus (aa 638-652) in complex with the PulS pilotin, a homology model was generated using Swissmodel based on the equivalent structure from Dickeya dadantii PDB $4 \mathrm{KOU}(>50 \%$ sequence identity for both chains). The map was low pass filtered to $8 \AA$ and the homology model initially fitted manually so that the PulS lipidated $\mathrm{N}$-terminus orientated towards the membrane. The Chimera Fit in Map function was then used for final positioning. Cross-validations were carried out as previously described ${ }^{10,56}$ using the auto-estimated B-factor sharpened map. Briefly, the PulD secretin model was displaced randomly by $0.2 \AA$ and then refined against a map reconstructed from one of the independent data halves (Half map 1). FSC curves were then calculated using the resulting model and Half map $1\left(\mathrm{FSC}_{\text {work }}\right)$. FSC curves were also 
calculated between this same model and another reconstruction generated from the other independent data half (Half Map 2 and $\mathrm{FSC}_{\text {free }}$ ). The similarity between $\mathrm{FSC}_{\text {work }}$ and $\mathrm{FSC}_{\text {free }}$ curves indicates an absence of overfitting within the PulD secretin model (Supplementary Fig. 1d). The final models were assessed using Molprobity ${ }^{57}$ and statistics outlined in Supplementary Table 1.

Reporting summary. Further information on research design is available in the Nature Research Reporting Summary linked to this article.

\section{Data availability}

3D cryo-EM density maps produced in this study have been deposited in the Electron Microscopy Data Bank with accession code EMD-0193. Atomic coordinates have been deposited in the Protein Data Bank (PDB) under accession code 6HCG.

Received: 14 October 2019; Accepted: 25 October 2019; Published online: 28 November 2019

\section{References}

1. Cianciotto, N. P. \& White, R. C. Expanding role of type II secretion in bacterial pathogenesis and beyond. Infect. Immun. 85, e00014-e00017 (2017).

2. Nivaskumar, M. \& Francetic, O. Type II secretion system: a magic beanstalk or a protein escalator. Biochim. Biophys. Acta 1843, 1568-1577 (2014).

3. Korotkov, K. V., Sandkvist, M. \& Hol, W. G. The type II secretion system: biogenesis, molecular architecture and mechanism. Nat. Rev. Microbiol. 10, 336-351 (2012).

4. McLaughlin, L. S., Haft, R. J. F. \& Forest, K. T. Structural insights into the Type II secretion nanomachine. Curr Opin Struct Biol 22, 208-216 (2012).

5. Guilvout, I., Chami, M., Engel, A., Pugsley, A. P. \& Bayan, N. Bacterial outer membrane secretin PulD assembles and inserts into the inner membrane in the absence of its pilotin. EMBO J. 25, 5241-5249 (2006).

6. Daefler, S., Guilvout, I., Hardie, K. R., Pugsley, A. P. \& Russel, M. The Cterminal domain of the secretin PulD contains the binding site for its cognate chaperone, PulS, and confers PulS dependence on pIVf1 function. Mol. Microbiol. 24, 465-475 (1997).

7. Yin, M., Yan, Z. F. \& Li, X. M. Structural insight into the assembly of the type II secretion system pilotin-secretin complex from enterotoxigenic Escherichia coli. Nat. Microbiol 3, 581-587 (2018).

8. Hay, I. D., Belousoff, M. J., Dunstan, R. A., Bamert, R. S. \& Lithgow, T. Structure and membrane topography of the Vibrio-type secretin complex from the type 2 secretion system of enteropathogenic Escherichia coli. J. Bacteriol. 200, 00521-17 (2018).

9. Hay, I. D., Belousoff, M. J. \& Lithgow, T. Structural basis of type 2 secretion system engagement between the inner and outer bacterial membranes. $m$ Bio $\mathbf{8}$, 01344-17 (2017).

10. Yan, Z. F., Yin, M., Xu, D. D., Zhu, Y. Q. \& Li, X. M. Structural insights into the secretin translocation channel in the type II secretion system. Nat. Struct. Mol. Biol. 24, 177-183 (2017).

11. Lallemand, M. et al. Dynamic interplay between the periplasmic and transmembrane domains of GspL and GspM in the type II secretion system. PLoS One 8, e79562 (2013).

12. Abendroth, J. et al. The three-dimensional structure of the cytoplasmic domains of EpsF from the type 2 secretion system of Vibrio cholerae. J. Struct. Biol. 166, 303-315 (2009).

13. Py, B., Loiseau, L. \& Barras, F. An inner membrane platform in the type II secretion machinery of Gram-negative bacteria. EMBO Rep. 2, 244-248 (2001).

14. Lu, C. et al. Hexamers of the type II secretion atpase gspe from Vibrio cholerae with increased atpase activity. Structure 21, 1707-1717 (2013).

15. Mancl, J. M., Black, W. P., Robinson, H., Yang, Z. M. \& Schubot, F. D. Crystal structure of a type IV pilus assembly ATPase: Insights into the molecular mechanism of pilb from thermus thermophilus. Structure 24, 1886-1897 (2016).

16. Satyshur, K. A. et al. Crystal structures of the pilus retraction motor PilT suggest large domain movements and subunit cooperation drive motility. Structure 15, 363-376 (2007).

17. Lu, C., Korotkov, K. V. \& Hol, W. G. J. Crystal structure of the full-length ATPase GspE from the Vibrio vulnificus type II secretion system in complex with the cytoplasmic domain of GspL. J. Struct. Biol. 187, 223-235 (2014).

18. Abendroth, J., Murphy, P., Sandkvist, M., Bagdasarian, M. \& Holl, W. G. J. The X-ray structure of the type II secretion system complex formed by the Nterminal domain of EpsE and the cytoplasmic domain of EpsL of Vibrio cholerae. J. Mol. Biol. 348, 845-855 (2005).
19. Arts, J. et al. Interaction domains in the Pseudomonas aeruginosa type II secretory apparatus component XcpS (GspF). Microbiology 153, 1582-1592 (2007).

20. Lopez-Castilla, A. et al. Structure of the calcium-dependent type 2 secretion pseudopilus. Nat. Microbiol 2, 1686-1695 (2017).

21. Sandkvist, M. Biology of type II secretion. Mol. Microbiol. 40, 271-283 (2001).

22. Korotkov, K. V. et al. Structural and functional studies on the interaction of GspC and GspD in the type II secretion system. PLoS Pathog. 7, e1002228 (2011).

23. Butt, R. H. \& Coorssen, J. R. Coomassie Blue as a near-infrared fluorescent stain: a systematic comparison with Sypro Ruby for in-gel protein detection. Mol. Cell. Proteom. 12, 3834-3850 (2013).

24. Luo, S., Wehr, N. B. \& Levine, R. L. Quantitation of protein on gels and blots by infrared fluorescence of Coomassie blue and Fast Green. Anal. Biochem. 350, 233-238 (2006).

25. Korotkov, K. V., Pardon, E., Steyaert, J. \& Hol, W. G. J. Crystal structure of the $\mathrm{N}$-terminal domain of the secretin GspD from ETEC determined with the assistance of a nanobody. Structure 17, 255-265 (2009).

26. Van der Meeren, R. et al. New insights into the assembly of bacterial secretins: structural studies of the periplasmic domain of XcpQ from Pseudomonas aeruginosa. J. Biol. Chem. 288, 1214-1225 (2013).

27. Douzi, B. et al. Unraveling the self-assembly of the Pseudomonas aeruginosa $\mathrm{XcpQ}$ secretin periplasmic domain provides new molecular insights into type II secretion system secreton architecture and dynamics. mBio 8, e01185-17 (2017).

28. Scheres, S. H. Processing of structurally heterogeneous cryo-EM data in RELION. Methods Enzymol. 579, 125-157 (2016).

29. Wang, X. H. et al. Cysteine scanning mutagenesis and disulfide mapping analysis of arrangement of $\mathrm{GspC}$ and GspD protomers within the type 2 secretion system. J. Biol. Chem. 287, 19082-19093 (2012).

30. Rehman, S., Gu, S., Shevchik, V. E. \& Pickersgill, R. W. Anatomy of secretin binding to the Dickeya dadantii type II secretion system pilotin. Acta Crystallogr. D. Biol. Crystallogr. 69, 1381-1386 (2013).

31. Dunstan, R. A. et al. Assembly of the type II secretion system such as found in Vibrio cholerae depends on the novel pilotin AspS. PLoS. Pathog. 9, e1003117 (2013).

32. Kastner, B. et al. GraFix: sample preparation for single-particle electron cryomicroscopy. Nat. Methods 5, 53-55 (2008).

33. Misic, A. M., Satyshur, K. A. \& Forest, K. T. P. aeruginosa PilT structures with and without nucleotide reveal a dynamic type IV pilus retraction motor. $J$. Mol. Biol. 400, 1011-1021 (2010).

34. Chang, Y. W. et al. Architecture of the type IVa pilus machine. Science 351, aad2001 (2016).

35. Collins, R. et al. Structural cycle of the Thermus thermophilus PilF ATPase: the powering of type IVa pilus assembly. Sci. Rep. 8, 14022 (2018).

36. Low, H. H. et al. Structure of a type IV secretion system. Nature 508, 550-553 (2014).

37. Michel-Souzy, S. et al. Direct interactions between the secreted effector and the T2SS components GspL and GspM reveal a new effector-sensing step during type 2 secretion. J. Biol. Chem. 293, 19441-19450 (2018).

38. Worrall, L. J. et al. Near-atomic-resolution cryo-EM analysis of the Salmonella T3S injectisome basal body. Nature 540, 597-601 (2016).

39. Gibson, D. G. et al. Enzymatic assembly of DNA molecules up to several hundred kilobases. Nat. Methods 6, 343-345 (2009).

40. Datsenko, K. A. \& Wanner, B. L. One-step inactivation of chromosomal genes in Escherichia coli K-12 using PCR products. Proc. Natl. Acad. Sci. U.S.A. 97, 6640-6645 (2000)

41. Nouwen, N. et al. Secretin PulD: association with pilotin PulS, structure, and ion-conducting channel formation. Proc. Natl. Acad. Sci. U. S. A. 96, 8173-8177 (1999).

42. Zheng, S. Q. et al. MotionCor2: anisotropic correction of beam-induced motion for improved cryo-electron microscopy. Nat. Methods 14, 331-332 (2017).

43. Zhang, K. Gctf: Real-time CTF determination and correction. J. Struct. Biol. 193, 1-12 (2016).

44. Scheres, S. H. RELION: implementation of a Bayesian approach to cryo-EM structure determination. J. Struct. Biol. 180, 519-530 (2012).

45. Orlova, S. S. Theory of three-dimensional reconstruction. 1. Conditions of a complete set of projections. Sov. phys., cryst. 20, 312-314 (1975).

46. Reichow, S. L., Korotkov, K. V., Hol, W. G. \& Gonen, T. Structure of the cholera toxin secretion channel in its closed state. Nat. Struct. Mol. Biol. 17, 1226-1232 (2010).

47. Rosenthal, P. B. \& Henderson, R. Optimal determination of particle orientation, absolute hand, and contrast loss in single-particle electron cryomicroscopy. J. Mol. Biol. 333, 721-745 (2003).

48. Jakobi, A. J., Wilmanns, M. \& Sachse, C. Model-based local density sharpening of cryo-EM maps. eLife 6, e27131 (2017).

49. van Heel, M., Harauz, G., Orlova, E. V., Schmidt, R. \& Schatz, M. A new generation of the IMAGIC image processing system. J. Struct. Biol. 116, 17-24 (1996). 
50. Frank, J. et al. SPIDER and WEB: Processing and visualization of images in 3D electron microscopy and related fields. J. Struct. Biol. 116, 190-199 (1996).

51. Zhang, Y. I-TASSER server for protein 3D structure prediction. BMC Bioinforma. 9, 40 (2008).

52. Waterhouse, A. et al. SWISS-MODEL: homology modelling of protein structures and complexes. Nucleic Acids Res. 46, W296-W303 (2018).

53. Pettersen, E. F. et al. UCSF Chimera-a visualization system for exploratory research and analysis. J. Comput. Chem. 25, 1605-1612 (2004).

54. Emsley, P., Lohkamp, B., Scott, W. G. \& Cowtan, K. Features and development of Coot. Acta Crystallogr. D. Biol. Crystallogr. 66, 486-501 (2010).

55. Adams, P. D. et al. PHENIX: a comprehensive Python-based system for macromolecular structure solution. Acta Crystallogr. D. Biol. Crystallogr. 66, 213-221 (2010).

56. Li, N. N. et al. Structure of the eukaryotic MCM complex at 3.8 angstrom. Nature 524, 186-191 (2015).

57. Chen, V. B. et al. MolProbity: all-atom structure validation for macromolecular crystallography. Acta Crystallogr. D. Biol. Crystallogr. 66, $12-21(2010)$

\section{Acknowledgements}

We thank eBIC for cryo-EM data collection support particularly Kyle Dent and Yuriy Chaban. Tillmann Pape and Paul Simpson for in-house EM support. Francesca Gubellini for gold labelling advice. Arjen Jakobi and Carsten Sachse for LocScale support. This work was funded by a Wellcome Trust Career Development Fellowship Enhancement Award (200074/Z/15/Z) to H.L.

\section{Author contributions}

A.C. and H.L. designed experiments. H.L. initially cloned and purified the Pul CDELMNS complex. A.C. and H.L. cloned and purified proteins, collected and processed data, and solved structure. A.C. built the OMC structure. A.C. and H.L. determined stoichiometry. A.C. undertook gold labelling. H.L. wrote the paper with contributions from A.C.

\section{Competing interests}

The authors declare no competing interests.

\section{Additional information}

Supplementary information is available for this paper at https://doi.org/10.1038/s41467019-13301-3.

Correspondence and requests for materials should be addressed to H.H.L.

Peer review information Nature Communications thanks the anonymous reviewers for their contribution to the peer review of this work

Reprints and permission information is available at http://www.nature.com/reprints

Publisher's note Springer Nature remains neutral with regard to jurisdictional claims in published maps and institutional affiliations.

(c) (i) Open Access This article is licensed under a Creative Common Attribution 4.0 International License, which permits use, sharing, adaptation, distribution and reproduction in any medium or format, as long as you give appropriate credit to the original author(s) and the source, provide a link to the Creative Commons license, and indicate if changes were made. The images or other third party material in this article are included in the article's Creative Commons license, unless indicated otherwise in a credit line to the material. If material is not included in the article's Creative Commons license and your intended use is not permitted by statutory regulation or exceeds the permitted use, you will need to obtain permission directly from the copyright holder. To view a copy of this license, visit http://creativecommons.org/ licenses/by/4.0/.

(C) The Author(s) 2019 